\title{
Treatment of osteosarcoma around the knee in skeletally immature patients
}

\author{
WEITAO YAO, QIQING CAI, JIAQIANG WANG and SONGTAO GAO
}

Bone and Soft Department, The Affiliated Cancer Hospital of Zhengzhou University, Zhengzhou, Henan 450000, P.R. China

Received April 27, 2016; Accepted June 29, 2017

DOI: $10.3892 / \mathrm{ol} .2017 .6903$

\begin{abstract}
Limb sparing surgery in growing young patients with malignant tumors is difficult as invasion of the physis by the tumor or surgical resection through the metaphysis may cause significant limb discrepancy following surgery. At present, hinged tumor prosthesis or biological reconstructions are the main methods following tumor resection in these patients. The aim of the present study was to assess different procedures for the treatment of osteosarcoma around knee joints in immature patients. A retrospective study of 56 patients $(<15$ years old, open physis) who had been treated for osteosarcoma around the knee joint between January 2007 and December 2015 was performed. Clinical data collected included patient demographics (age at diagnosis, sex and date of diagnosis), tumor characteristics [location, Enneking stage and subtype on magnetic resonance imaging (MRI)], treatment (response to neoadjuvant chemotherapy and type of primary surgery) and clinical outcomes (limb function, discrepancy and overall survival). The median age at the time of diagnosis was 12.14 years (range, 3-15 years). There were 32 male patients $(57.1 \%)$. A total of $41(82 \%)$ tumors were located at the distal femur, and $15(18 \%)$ at the proximal tibia. A total of $49(87.5 \%)$ patients were diagnosed with stage IIB tumors, and $7(12.5 \%)$ had stage III, according to the Enneking stage classification. Different surgical methods, including amputation, rotation-plasty, endoprosthesis and biological instructions (e.g., allograft) were performed according to MRI type classification. During follow-up, 21 patients (37.5\%) succumbed to disease. The Musculoskeletal Tumor Society score ranged from excellent to fair functional result. Recurrence (2 cases, $16.67 \%)$ and infection (2, cases, $16.67 \%$ ) were the main complications following endoprosthesis replacement, while delayed union (12 cases, $57.14 \%$ ) and fracture (3 cases, 14.29\%) were the main causes for biological reconstructions. Limb-length
\end{abstract}

Correspondence to: Dr Weitao Yao, Bone and Soft Department, The Affiliated Cancer Hospital of Zhengzhou University, 127 Dong Ming Road, Zhengzhou, Henan 450000, P.R. China

E-mail:ywtwhm@163.com

Key words: limb saving surgery, osteosarcoma, immature patient, endoprosthesis, biological construction, overall survival discrepancy ranged from $0-10 \mathrm{~cm}$ in limb-saving surgery. The overall survival rate was $57.66 \%$ with different cohorts in Enneking stages IIB and III, with or without involvement of the physis and different cycles of chemotherapy. Results of the present study indicated that different limb saving surgeries, including epiphysis/physis preservation with biological construction in patients with MRI types I to III and endoprosthetic/osteoarticular reconstruction in patients with MRI types IV and V, are useful in the management of osteosarcoma in growing young patients with proper surgery indications, and knee joint function was maintained with acceptable complications including limb discrepancy, delayed union, infection, recurrence and fracture.

\section{Introduction}

Osteosarcoma is the most common malignant bone tumor in children, adolescents, and young adults. The occurring rate was 4.0 (3.5-4.6) for 0-14 year-old children per year per million of people for males and females and from all ethnicities (1). Tumors arise primarily on the metaphysis, which is near the growth plate and gradually invade the epiphysis and eventually the whole joint space (2). The distal femur and proximal tibia are the most common sites for osteosarcomas, and the epiphyses of the distal femur and proximal tibia contribute $\sim 35$ and $30 \%$ to the growth of the lower extremity, respectively (3).

With rising survival rates following chemotherapy, limb salvage surgery is increasingly becoming the standard of care for the majority of malignant neoplasms affecting the extremities $(4,5)$. Segmental bone loss following tumor resection requires prosthesis reconstruction in most adult patients, but may be difficult in skeletally immature patients due to the necessity to preserve the joint function maximally and maintain good limb function (6). This is a challenging situation for surgeons treating patients following epiphysis resection and limb reconstruction with the most suitable procedure in order for the least length discrepancy compared with the ongoing growth of the contralateral limb.

The growth plate has a key role in limb growth (7). Assessment of the relationship of the growth plate and tumor helps surgeons determine surgical options based on the involvement of this region and the extent of the tumor. This will have implications on the clinical result by potentially affecting limb length and/or the function of the involved. Therefore, clear images are the first step in treating tumors in immature 
patients. In recent years, the extension of these tumors has been determined through preoperative diagnostic imaging techniques, primarily via magnetic resonance imaging (MRI) (8). The extension of the tumor can be accurately evaluated on T1-weighted, T2-weighted, and Gd-enhanced T1-weighted MRI images in coronal, sagittal and axial planes. According to the involved anatomical sites on MRI, Kumta et al (9) classified the location and extension of osteosarcoma in bone into five subtypes as follows: Type 1 , the tumor is located $>2 \mathrm{~cm}$ from the epiphyseal cartilage; type II, the tumor is located within $\leq 2 \mathrm{~cm}$ of the epiphyseal cartilage; type III, the tumor extends to or beyond the epiphyseal cartilage, but $>1 \mathrm{~cm}$ of epiphyseal tissue is retained; type IV, the tumor breaches the physes and extends to the subchondral region but does not breach the articular surface; and type V, the tumor breaches the articular surface and involves the adjacent joint.

In the present study, osteosarcoma in immature patients (open physis and age, $<15$ years) around the knee joint was classified into five types according to the classification system as described by Kumta et al (9) using preoperative MRI, and limb reconstruction or amputation methods were performed following wide resection. The goals of the present study were to assess: i) Characteristics of osteosarcoma in immature patients, ii) different MRI types with adequate surgical methods, iii) the benefits and complications of different surgical methods, and iv) overall survival (OS) rate and factors that affect OS.

\section{Patients and methods}

In the present study, the authors performed a retrospective study of the characteristics and outcomes in immature patients diagnosed with osteosarcoma around the knee joint treated at the Henan Cancer Hospital (Zhengzhou, China).

Patients. The cohort consisted of 56 patients (age, $<15$ years) diagnosed with open physis with osteosarcoma at the distal femur and proximal tibia between January 2007 and December 2015 that were treated at the Henan Cancer Hospital. Ethical approval was obtained from the Medical Ethics Committee of the Henan Cancer Hospital. Written informed consent was obtained from the patients' legal guardians for publication of the present report and accompanying images. The following information was collected: i) Patient demographics including age at diagnosis, sex and date of diagnosis; ii) tumor characteristics including location, Enneking stage (10), subtype on MRI and histology; iii) treatment including response to neoadjuvant chemotherapy, type of primary surgery, postoperative treatment and adverse effects; iv) clinical outcomes, including limb discrepancy, OS, disease-free survival (DFS), event-free survival (EFS) and predictive factors that are associated with survival.

Treatment. Chemotherapy was utilized in the neoadjuvant and adjuvant settings. The chemotherapy regimens, specifically dose, were based on body surface area of the patients. The chemotherapy included combinations of high doses of methotrexate (Jiangsu Hengrui Medicine Co., Ltd., Linyungang, China), carboplatin (Corden Pharma Latina S.P.A., Sermoneta, Italy) (11), doxorubicin/pirarubicin (Shenzhen Main Luck
Pharmaceuticals Inc., Shenzhen, China) $(12,13)$ and ifosfamide (Jiangsu Hengrui Medicine Co., Ltd.). Each patient was administered with all the agents. Tumor necrosis percentage (TNP) was accessed by pathology following operation. The percentage area of necrosis was calculated in at least 4 continuous slides of each spice and the sum was used to give a percentage of necrosis of the whole tumor under a light microscope (magnification, x100).

Of the 56 patients, 4 abandoned the treatment while the remaining 52 patients underwent surgery for local control and had negative surgical margins as confirmed by pathology. The type of surgery including limb salvage surgeries, such as tumor hinged prosthesis replacement (TPR, Chun Li Co., Ltd., Beijing, China), osteoarticular allograft replacement (OAR), inactivated auto-osteoarticular replacement (IOR), intercalary allograft replacement (IAR), autogenous bone replacement (ABR), amputation (AP), rotation-plasty (RP) was based on the extent of disease, involvement of neurovascular bundle, and appraisal for best limb functionality following surgical resection. All structural allograft bones were procured according to the protocol of the Chinese Association of Tissue Banks (14) and obtained from the bone bank. Trans-meta/epiphyseal osteotomy (15) or physeal distraction (16) was used to preserve the uninvolved physis (PUP) in certain patients. Arthrodesis reconstruction was not performed in any of these patients.

Follow-up. Oncology follow-up was performed at three monthly intervals for the first two years and six monthly intervals until 5 years. Bone healing and implantation was assessed using antero-posterior and lateral radiographs. The patients were checked regularly to detect pulmonary metastases with computed tomography (CT) scan. Functional results were assessed using the Musculoskeletal Tumor Society (MSTS) score (17) at the last follow-up visit. Grading of MSTS score is as follows: $\leq 23$, excellent functional result; 15-22, good result; $8-14$, fair result and $<8$, poor result. Joint range of motion, strength, muscular atrophy and lower limb length discrepancy were also assessed at the follow-up visits. Final limb-length discrepancy was measured by teleroentgenogram showing the entire length of the legs on one film. The follow-up was mean of 21.66 months. At the end of follow-up, the median age of the patients was 14.31 years (range, 3-23 years), and 18 (32.14\%) patients reached skeletal maturity.

Statistical analysis. The primary focus of the present analysis was OS, EFS and DFS. OS was calculated from the date of diagnosis to the date of mortality or most recent follow-up examination. The survival curves were calculated using the Kaplan-Meier estimate with $95 \%$ confidence interval. The differences of survival curves were assessed using the log-rank test. Adjusted estimates were obtained from proportional hazards models with sex, age, clinical Enneking stage (10), MRI type and surgical method included as covariates. MSTS scores and differences in limb length of different surgeries were compared using one-way analysis of variance with the least significant difference by comparing means for continuous variables. The associations between the age at diagnosis and tumor invasion of the physis, response to treatment and survival were compared using the Mann-Whitney test. $\mathrm{P}<0.05$ was considered to indicate a statistically significant difference. 
Table I. Demographic and clinical characteristics of 56 patients.

\begin{tabular}{lcc}
\hline Patient demographics & $\mathrm{n}$ & Cohort $(\%)$ \\
\hline Age at diagnosis, years & & \\
0-3 & 1 & 1.8 \\
$3-6$ & 1 & 1.8 \\
$6-9$ & 9 & 16.1 \\
$9-12$ & 12 & 21.4 \\
12-15 & 33 & 58.9 \\
Mean age, years (range) & $12.14(3-15)$ & \\
Sex & & \\
Female & 24 & 42.9 \\
Male & 32 & 57.1 \\
Tumor site & & \\
Left distal femur & 27 & 48.2 \\
Right distal femur & 14 & 25.0 \\
Left proximal tibia & 5 & 17.9 \\
Right proximal tibia & 10 & \\
Clinical Enneking stage & & 12.5 \\
IIB & 49 & \\
III & 7 & \\
\hline
\end{tabular}

SPSS software (version 11.5; SPSS, Inc., Chicago, IL, USA) was used.

\section{Results}

Patient demographics. A total of 56 patients (age, $<15$ years) with diagnosis of osteosarcoma around the knee joint were involved in the present study. The complete demographics and clinical characteristics are shown in Table I. The median age, at the time of diagnosis, was 12.14 years (range, 3-15 years). There were 32 male patients $(57.1 \%)$. Tumor location was as follows: $41(82 \%)$ at the distal femur and $15(18 \%)$ at the proximal tibia. High grade, conventional osteosarcoma was diagnosed in all patients. A total of $49(87.5 \%)$ patients had stage IIB tumors and 7 (12.5\%) had stage III tumors, according to the Enneking stage classification.

Treatment. Therapy methods are shown in Fig. 1 and were based on MRI findings prior to operation. There were 45 $(80.36 \%)$ limb growth plates and epiphyses closed to or invaded by tumor from MRI type III to V. In 7 patients with stage III, a total of 4 patients (1 with type III and 2 with type IV) abandoned additional interventions, 3 with lung metastases ( 2 with type $\mathrm{V}$ and 1 with type IV) underwent debulking surgery, which comprised amputations (according to the patient's choice) and 2-6 cycles of chemotherapy. In other patients with stage IIB, a total of 4 patients (1 with type V, 2 with type IV and 1 with type III) underwent amputations due to massive tumor sizes and insensitivity to chemotherapy, 3 patients ( 2 with type III and 1 with type IV) underwent rotation-plasty (Fig. 2A), and the remaining 42 patients underwent various limb saving surgeries. A patient each with type III, IV and V was treated by semi

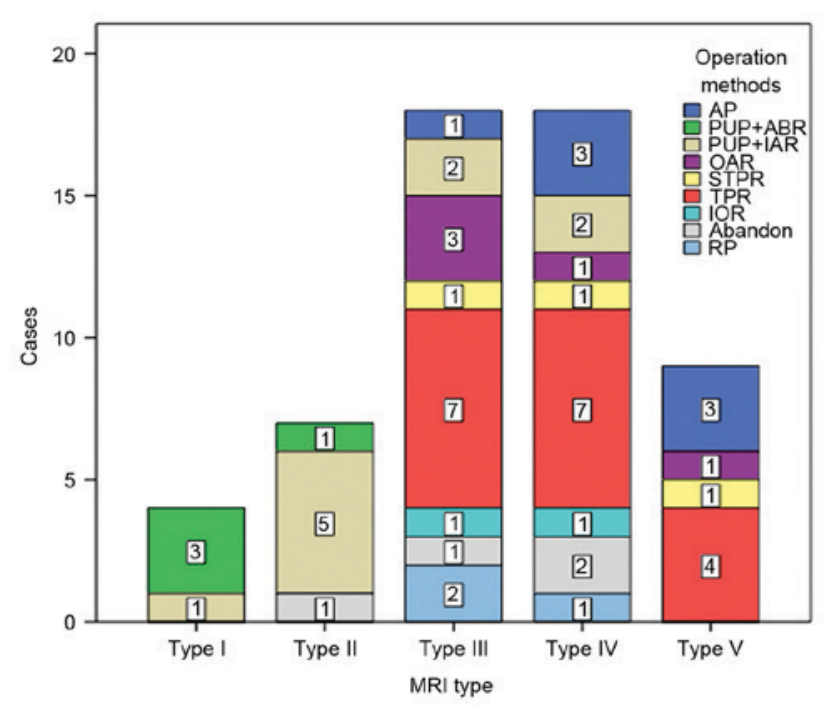

Figure 1. Treatment methods classified according to MRI types in 56 patients. AP, amputation; PUP, preserve the uninvolved physis; ABR, inactive autogenous bone replacement; IAR, intercalary allograft replacement; OAR, osteoarticular allograft replacement; IOR, inactivated auto-osteoarticular replacement; TPR, tumor prosthesis replacement; STPR, semi tumor prosthesis replacement; RP, rotation-plasty; MRI, magnetic resonance imaging.

femur TPR, to preserve the adjacent semi joint and growing physis (Fig. 2B). TPR is widely adapted for tumors growing close to joints $(18,19)$. In the present study, a total of 18 (42.86\%) cases $(7,7,4$ with types III, IV, V, respectively) accepted this method (Fig. 2C). A total of 21 patients had biological constructions as follows: 4 patients with type 1 underwent resection of the tumor by transverse osteotomy at the metaphysis with retention of the physeal plate and a small portion of the adjacent metaphysis. Subsequently, IAR was used to reconstruct the defect in 1 patient, and inactive (anhydrous alcohol, $40 \mathrm{~min}$ ) autogenous bone replacement (ABR) was performed in 3 patients. A total of 6 patients with type II preserved PUP by physeal distraction, which was then reconstructed by IAR (Fig. 2D) in 5 patients and ABR in 1 patient (Fig. 2E). Due to the tumor invasion of the physis in types III-V, 4 cases underwent intraepiphyseal resection and reconstructed by IAR ( 2 with type III and IV). A total of 5 patients underwent OAR ( 3 with type III, 1 with type IV and 1 with type V; Fig. 2F). A total of 2 (1 with type III and 1 with type IV) underwent inactivated auto-osteoarticular replacement (IOR; inactivation with anhydrous alcohol for $40 \mathrm{~min})$.

Follow-up. A total of 21 patients (37.5\%) succumbed to disease, including local recurrence in 4 patients who underwent amputation at 6-39 months postoperatively. A total of 17 patients succumbed to pulmonary metastases. A total of 3 patients went through one or more times of surgical resection of pulmonary recurrence and 1 patient survived for 42 months at the end of follow-up. The mean time of mortality was 13.14 months following diagnosis (Table II). All patients experienced mild to severe myelosuppression but without renal or cardiac toxicity.

According to post-operative pathological examination, the TNP was $>90 \%$ in $32(61.54 \%)$ patients, $80-90 \%$ in 17 cases 
Table II. Outcomes and complications of different operation methods.

\begin{tabular}{|c|c|c|c|c|c|c|c|c|}
\hline \multirow{2}{*}{$\begin{array}{l}\text { Operation } \\
\text { methods }\end{array}$} & \multirow[b]{2}{*}{ Cases, $\mathrm{n}$} & \multirow{2}{*}{$\begin{array}{l}\text { MSTS } 93 \\
\text { score }^{\mathrm{a}}\end{array}$} & \multirow{2}{*}{$\begin{array}{l}\text { Limb length } \\
\text { discrepancy, } \mathrm{cm}^{\mathrm{a}}\end{array}$} & \multicolumn{5}{|c|}{ Complications, $\mathrm{n}$} \\
\hline & & & & Recurrence & Metastasis & Infection & Nonunion & Fracture \\
\hline TPR & 18 & $24.89 \pm 2.65$ & $2.64 \pm 1.91$ & 2 & 5 & 2 & 0 & 0 \\
\hline STPR & 3 & $23.33 \pm 2.08$ & $2.50 \pm 0.71$ & 0 & 1 & 0 & 0 & 0 \\
\hline PUP+IAR & 10 & $23.40 \pm 6.33$ & $2.71 \pm 1.25$ & 1 & 2 & 1 & 5 & 1 \\
\hline PUP+ABR & 4 & $25.25 \pm 3.59$ & $3.33 \pm 3.21$ & 1 & 0 & 0 & 2 & 1 \\
\hline OAR & 5 & $21.40 \pm 3.85$ & $6.00 \pm 1.41$ & 0 & 2 & 1 & 3 & 1 \\
\hline IOR & 2 & $12.50 \pm 3.54^{\mathrm{b}}$ & $6.00 \pm 3.61$ & 0 & 1 & 0 & 2 & 0 \\
\hline $\mathrm{RP}$ & 3 & $24.67 \pm 1.53$ & & 0 & 1 & 0 & 0 & 0 \\
\hline $\mathrm{AP}$ & 7 & $14.50 \pm 2.65^{\mathrm{b}}$ & & 0 & 5 & 0 & 0 & 0 \\
\hline
\end{tabular}

${ }^{\mathrm{a}}$ Mean \pm standard deviation; ${ }^{\mathrm{b}} \mathrm{P}<0.05$. AP, amputation; PUP, preserve the uninvolved physis; ABR, inactive autogenous bone replacement; IAR, intercalary allograft replacement; OAR, osteoarticular allograft replacement; IOR, inactivated auto-osteoarticular replacement; TPR, tumor prostheses replacement; STPR, semi tumor prostheses replacement; RP, rotation-plasty; MSTS, Musculoskeletal Tumor Society score.
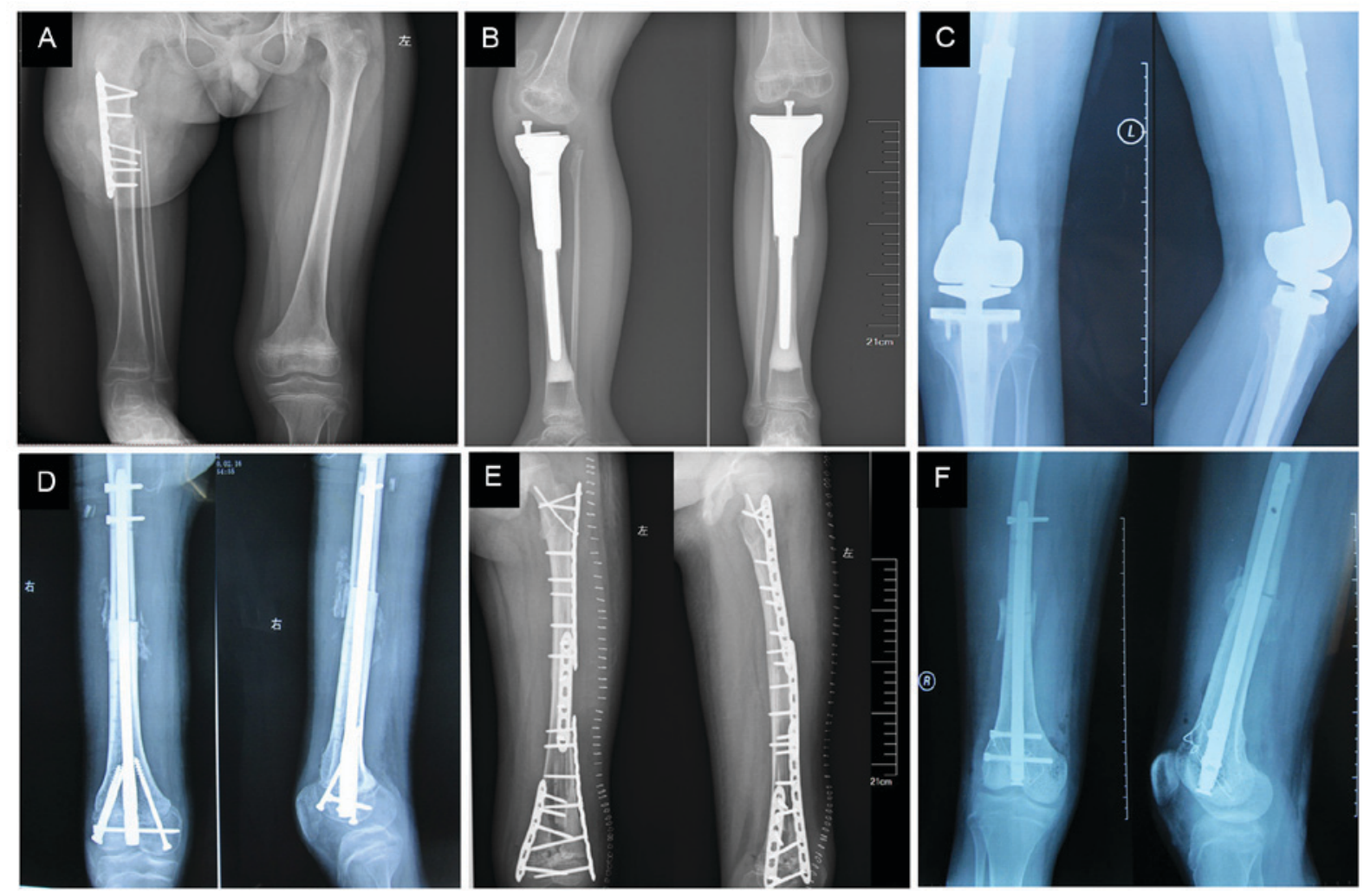

Figure 2. Different surgical methods performed in immature osteosarcoma patients. (A) Rotation-plasty following distal femur tumor resection in a 9 year-old boy. (B) Proximal tibia tumor resection of a 9 year-old girl. (C) Tumor prosthesis replacement of a 12 year-old boy. (D) PUP and intercalary allograft replacement following distal femur tumor resection of a 15 year-old boy. (E) PUP and inactive autogenous bone replacement following distal femur (almost entire diaphysis) tumor resection of a 9 year-old girl. (F) Osteoarticular allograft replacement following distal femur tumor resection of a 14 year-old boy. PUP, preservation of the uninvolved physis.

$(32.69 \%)$ and $<80 \%$ in 3 cases $(5.77 \%)$. There were no significant differences in age at diagnosis and tumor invasion of the physis $(\mathrm{P}=0.705)$, but differences in response to treatment and survival were significant $(\mathrm{P}<0.0001)$. Fig. 3 depicts follow-up images of patients.

In the present study, excellent functional result according to MSTS score was achieved in the TPR, PUP plus ABR and PR groups. Good result was achieved in the semi tumor prosthesis replacement (STPR, Fig. 3A), PUP plus IAR and OAR groups. Fair result was achieved in the AP and IOR groups. In the IOR group, two 6-year-old patients without suitable osteoarticular allograft had restrictions in recreational activities for 6-12 months due to absorption and nonunion of inactivated osteoarticular. 

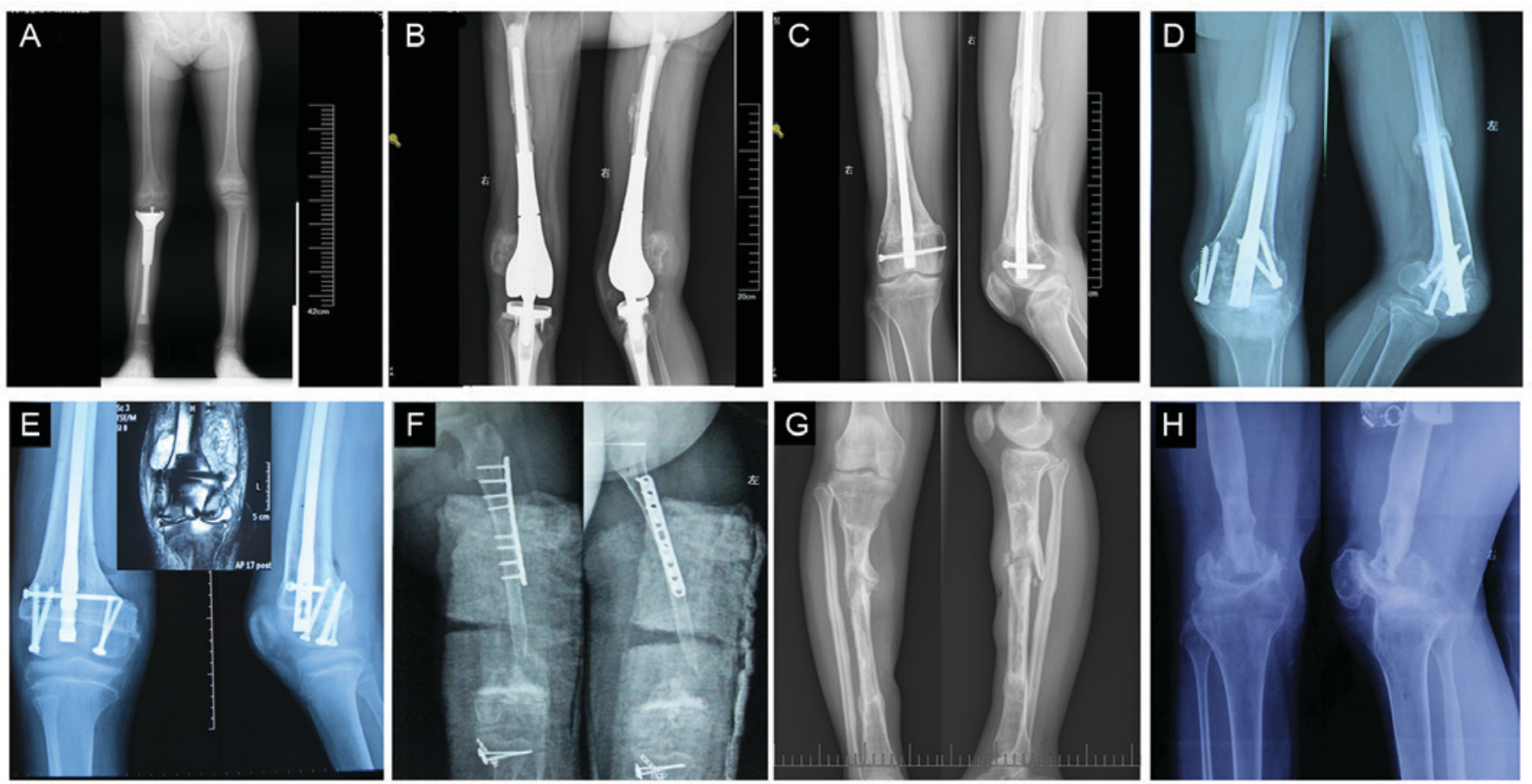

Figure 3. Follow-up of immature osteosarcoma patients. (A) Limb length discrepancy and knee joint instability following semi tumor prostheses replacement at 36 months post-treatment in a 9 year-old girl. (B) Recurrence occurred at 12 months following tumor prosthesis replacement in a 9 year-old girl. (C) Diaphysis nonunion occurred at 50 months following PUP and IAR in a 15 year-old boy. (D) Metaphyseal nonunion and dislocation at 24 months following PUP and IAR in a 14 year-old girl. (E) Recurrence occurred at 12 months following PUP by transverse osteotomy at the metaphysis in MRI type III in a 12 year-old boy (MRI indicated soft tumor around the metaphysis). (F) Bone absorption and nonunion occurred at the metaphysis of inactivated auto-osteoarticular replacement at 12 months in a 9 year-old girl. (G) A total of 10 months after all screws and plates had been removed, fracture occurred 60 months following PUP and inactive autogenous bone replacement in a 14 year-old girl. $(\mathrm{H})$ A total of 96 months following osteoarticular allograft replacement, metaphyseal fracture and knee joint degeneration occurred in a 14 year-old boy. IAR, intercalary allograft replacement; PUP, preservation of the uninvolved physis.

This caused the low MSTS score and dysfunction of the knee joint (Table II).

Recurrence ( 2 cases, 16.67\%) and infection ( 2 cases, $16.67 \%)$ were the main complications in the TPR group (Table. II and Fig. 3B). Patients with local tumor recurrence underwent amputations. Prosthesis was removed in one patient who developed deep infection, a temporary cement spacer was implanted and antibiotics of Cefotiam were administered. After 6 months of treatment and observation, another prosthesis was replaced. The other patient with delayed infected prostheses (occurred at 24 months following surgery) underwent immediate amputation. During the follow-up, loosening or fractures of prostheses were not seen in these patients. In patients of biological reconstructions, the main complications were delayed union (12 cases, 57.14\%) and fracture ( 3 cases, $14.29 \%)$. Delayed union occurred at the diaphyseal in 7, at metaphyseal in 3 case and at both places in 2 cases (Fig. 3C and D). The delayed union was treated by autologous iliac bone replantation and/or replacement of internal fixation, which eventually resulted in union in 10 cases $(90.48 \%)$ at the host donor junction. All the fractures occurred when the internal fixation had been taken out 48-72 months following tumor resection (Fig. 3G). Patients refused additional operation without pain of the involved limb.

Limb-length discrepancies $(0-3 \mathrm{~cm})$ were observed in four patients in MRI type I with both physes preserved caused by the growth plate partly injured by internal fixations. In the remaining 38 limb-saving patients, limb-length discrepancy developed as a result of loss of one or two physes (Fig. 3A).
At final follow-up, the mean shortage was $3.32 \mathrm{~cm}$ (range, $1-10 \mathrm{~cm}$ ). There was no asociation between surgical methods, age at the time of diagnosis with limb discrepancies $(\mathrm{P}>0.05)$. A total of 18 patients had no discernible limp, 20 cases had a minor cosmetic limp and 4 cases had a major cosmetic limp.

Survival. The OS rate was $57.66 \%$ in 56 patients (Fig. 4A), the 2 and 5 year DFS was 48.21 and $10.71 \%$ respectively. The EFS of 1, 2 and 3 years was 85.02, 60.27 and 57.80\%, respectively. The OS rates were 67.01 and $0 \%$ for patients at Enneking stages IIB and III (Fig. 4B), respectively, and 86.67 and $44.97 \%$ for patients with and without physis (Fig. 4C), respectively. In patients with 6 and 5 cycles of chemotherapy, the OS rates were 82.17 and $33.33 \%$, respectively (Fig. 4D; $\mathrm{P}<0.05)$. There were no statistically significant differences in sex, age, surgical method and tumor location in relation to OS.

\section{Discussion}

In mature patients, prosthesis replacement is the main method for limb salvage surgery of malignant bone tumor (20). However, it is a controversial issue in patients with an immature skeletal age. At present, a variety of procedures have been used in these young patients, including prostheses, biological reconstruction, arthrodesis, rotation-plasty (21-23) and amputations. Children who undergo limb-sparing surgery of the lower limbs will face various problems postoperatively as they grow. In particular, limb-length discrepancies and loosening 

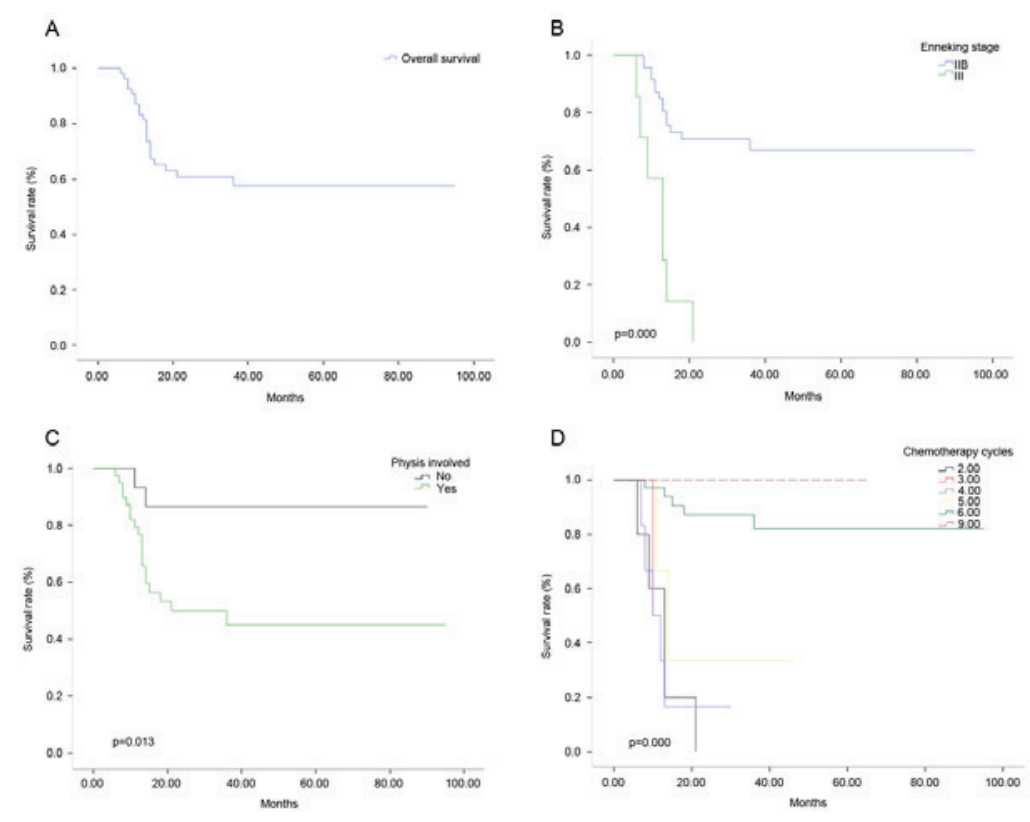

Figure 4. Overall survival rate with different hazards models. (A) Graph indicating the OS rate of 56 patients. (B) Graph showing different OS rates in patients at Enneking stages IIB and III. (C) Graph showing different OS rates in patients with and without physis. (D) Graph showing different OS rates in patients with 6 and 5 cycles of chemotherapy. OS, overall survival.

involving the prosthesis can cause serious limb dysfunction $(24,25)$.

Ablative surgery (amputation or rotation-plasty) was performed in patients with Enneking stage III disease (with lung metastasis and debulking surgery), local recurrence, extensive sarcoma and in those who were unresponsive to chemotherapy or were young ( $<9$ years old). Amputation can leave cosmetic, emotional and functional defects. In the present study, amputation was performed in 7 patients. Rotation-plasty permits the concurrent correction of limb-length discrepancies (26). Limb reconstruction is a good alternative to prosthetic limb with excellent function in young patients with a short recovery time, normal knee movement and no adverse events during follow-up, however for many patients there might be emotional and cosmetic concerns (27). In the present study, only three patients accepted rotation-plasty.

Hinged tumor prosthesis was used in immature patients (18 cases) in limb sparing surgery, because it fills the defect and immediately restores knee joint function and limb biomechanical stability. In the present cohort, 45 limb growth plates and epiphyses were invaded by tumor, and resection of tumor resulted in the loss of a significant portion of the joint surface.

Tumor prosthesis may be considered as one of the most convenient reconstructive options $(20,21)$. At present, epiphysis non-invasive expandable prosthesis is not commonly used in China due to high price, scarce lengthening equipment and high rate of complications, including distracted neurovascular injuries, deep infection and aseptic loosening of implantation $(25,28)$. Meanwhile, reconstruction with prosthesis compromises the growing physis. The growth physis of the segment is compromised by the tumor, and the physis of the opposite side of the joint may be physiologically altered in its growing potential by the intra-medullary stem perforation (29). This may cause inevitable limb discrepancy particularly in young children (age, $<9$ years). Therefore, a remodeled prosthesis with a smaller diameter $(<10 \mathrm{~mm})$ for intra-medullary stem was used in the present study, and the healthy part of the epiphysis was preserved (Fig. 2C). The involved limb with semi tibia prosthesis was also reconstructed (hemiarthroplasty) in 3 cases (Fig. 2B), as only the growth physis of the segment compromised by the tumor was sacrificed and the unaffected opposing joint cartilage was retained. Hemiarthroplasty resulted in multi-directional instability and limited the movement of knee joint during the follow-up. The limb length discrepancy ranged from 1 to $6 \mathrm{~cm}$ (average, $2.60 \mathrm{~cm}$ ) in TPR and STPR groups. In the two recurrences in the TPR group, huge tumor size (diameter, $>10 \mathrm{~cm}$ ), reduced sensitivity to chemotherapy (tumor necrosis rate $<90 \%$ ) and an inadequate resection margin were the main reasons of relapse.

In the long run, prosthesis may result in high rates of mechanical complications and limited articular function. In addition, as surviving patients have long life expectancy, it is very difficult for any prosthetic reconstruction to achieve durability during this time (30).

Compared with prosthesis replacement, biologic reconstructions require graft material to incorporate within the host. Once the graft bone has been substituted by autologous bone, a lower complication rate than prosthesis over time follows (31). Therefore, massive bone graft is commonly used in growing patients with long life expectations. Therefore, different resection and reconstruction methods were adopted according to the MRI image classification obtained prior to the operation (9).

In the present study, when a safe margin was present between the tumor and the growth plate and epiphysis, transverse osteotomy at the metaphysis was performed in patients with MRI type I as described by Kumta et al (9). Physeal distraction was performed in patients with MRI type II to preserve the joint surface and maintain joint function as described 
by Cañadell et al (16). Once safe margins were confirmed during operation by histological examination, replacement was applied by inactive autogenous bone or intercalary allograft. In cases with MRI type III, as the tumors are intact with the growth plate, intra-epiphyseal resection or osteoarticular replacement is used as an alternative to endoprosthetic reconstruction (16). However, care must be taken when determining surgical margins, and the tumor should not cross the growth plate for intra-epiphyseal resection (32). In the present study, although safe epiphyseal resection margins have been confirmed by pathology, 2 cases in the cohort relapsed within 6-12 months during follow-up (Fig. 3E, Table II). In cases with MRI type IV and V, osteoarticular allograft replacement was the preferred choice of treatment due to the invasion of growth plate and epiphysis by tumor cells as previously described by Kumta et al (9). In osteoarticular reconstructions in our study, the remaining ligaments were reattached to the corresponding allograft or inactive tissues by a direct lateral-lateral interval suture to improve stability. The host meniscus was reattached to the osteoarticular allograft, and both horn insertions and the articular capsule were sutured. The cruciate ligament of allograft or inactive tissues were inserted and fixed to host bone (33).

In the present study, the results indicated that preservation of the PUP with adequate margins and biological reconstruction in MRI types I, II and III may be an alternative to endoprosthetic reconstruction. The clinical results from PUP and endoprosthetic reconstruction indicated equal MSTS scores and knee joint function. As the patients' own joint cartilage and stabilizing structures, and the potential for continued axial growth can be retained, permanent curative effect can be acquired (34). The limb length discrepancy was $1-10 \mathrm{~cm}$ (average, $2.83 \mathrm{~cm}$ ) in the PUP plus IAR and PUP plus ABR groups in the present study. Discrepancy in the current cohort was not treated, as the normal life of the patients was not seriously affected.

In the present study, a better functional result was observed for endoprosthetic replacement than osteoarticular reconstruction in patients with MRI types IV and V. Due to the corrosion of joint fluid and long time restriction of movement, evident bone absorption and knee joint stiffness can be observed in the majority of osteoarticular replacement patients in the present study. Joint instability, degeneration of cartilage and metaphyseal fractures were observed in the patients in the present study (Fig. 3F and H), consistent with the findings of DeGroot et al (33). Joint instability, degeneration of cartilage and metaphyseal fractures caused lower MSTS scores and reduced knee joint function compared with prosthesis replacement. The limb length discrepancy ranged from 4-9 cm (average, $6.17 \mathrm{~cm}$ ) in the OAR and IOR groups.

In the present study, the results indicated that infections, particularly deep infections, which is a serious impediment and may require an amputation, are the main complications in prosthetic reconstruction. However, delayed or nonunion and fractures are the primary complications in biological constructions. The delayed union can be identified by progressive, massive absorption of the graft at the bone-graft junction, as the replaced allograft or inactive autologous bone lacks adequate blood supply. The treatment involves additional surgery with new autograft and changed fixation. To accelerate bone healing, a part of the intramedullary locked screws is usually removed 12 months following surgery to obtain a dynamic compressive force on the fracture surface, which can result in bony union in some cases (35).

The ultimate non-union rate in the present study was $9.52 \%$, which is similar to the rate reported in other studies $(36,37)$. In osteoarticular reconstructions, progressive articular degeneration was observed in the majority of patients as early as 3-5 years following implantation, resulting in narrow joint space and pain, decreased function of involved joint (Fig. 3H).

A number of factors have been reported to affect the clinical effect and patient survival rate of osteosarcoma. Faisham et al (38) analyzed 163 patients with osteosarcoma with an average age of 19 years (range, 6-59 years). It was reported that the OS rate in patients who completed chemotherapy and surgery $(n=117)$ was $72 \%$ at 2 years and $44 \%$ at 5 years post-treatment. The factors that affected survival rate were surgery methods (limb salvage prior to amputation) and the presence of lung metastasis. Ayerza et al (39) retrospectively reviewed 251 patients with high-grade osteosarcoma from 1980 to 1989 and reported higher rates of limb salvage treatment and survival, with a lower incidence of secondary amputation occurring with the use of chemotherapy. In the present study, the factors affecting overall survival rate included clinical Enneking stage, involvement of the growth plate, and cycles of chemotherapy.

However, individualized surgical procedures were performed on a limited number of 56 patients, and it would therefore be difficult to compare substantially different techniques with the same surgeon. Another limitation is a relatively short follow-up period (range, 2-95 months; average, 21.66 months) and that only some of the patients (18 cases, $32.14 \%$ ) reached their skeletal maturity at the last follow-up. Therefore, it was not possible to establish any final limb-length discrepancy. A longer follow-up is necessary to establish long-term survival of the different reconstructions and final limb-length discrepancies.

Different limb surgeries, including epiphysis/physis preservation with biological construction in MRI types I to III, endoprosthetic/osteoarticular reconstruction in MRI types IV and V, are useful in the management of osteosarcoma in growing young patients with proper surgery indications, and maintains knee joint function with acceptable complications including limb discrepancy, delayed union, infection, recurrence and fracture.

\section{References}

1. Ottaviani $\mathrm{G}$ and Jaffe N: The epidemiology of osteosarcoma. Cancer Treat Res 152: 3-13, 2009.

2. Chen Y, Yu XC, Xu SF, Xu M and Song RX: Impacts of tumor location, nature and bone destruction of extremity osteosarcoma on selection of limb salvage operative procedure. Orthop Surg 8: 139-149, 2016.

3. Haynes K, Tyner C and Williams PD: Repiphysis prosthesis for limb preservation in pediatric patients with bone cancer: A literature review. Orthop Nurs 32: 81-86, 2013.

4. Kudawara I, Aoki Y, Ueda T, Araki N, Naka N, Nakanishi H, Matsumine A, Ieguchi M, Mori S, Myoui A, et al: Neoadjuvant and adjuvant chemotherapy with high-dose ifosfamide, doxorubicin, cisplatin and high-dose methotrexate in non-metastatic osteosarcoma of the extremities: A phase II trial in Japan. J Chemother 25: 41-48, 2013. 
5. Hegyi M, Semsei AF, Jakab Z, Antal I, Kiss J, Szendroi M, Csoka M and Kovacs G: Good prognosis of localized osteosarcoma in young patients treated with limb-salvage surgery and chemotherapy. Pediatr Blood Cancer 57: 415-422, 2011.

6. Eleutério SJ, Senerchia AA, Almeida MT, Da Costa CM, Lustosa D, Calheiros LM, Barreto JH, Brunetto AL, Macedo CR and Petrilli AS: Osteosarcoma in patients younger than 12 years old without metastases have similar prognosis as adolescent and young adults. Pediatr Blood Cancer 62: 1209-1213, 2015.

7. Ruzbarsky JJ, Goodbody C and Dodwell E: Closing the growth plate: A review of indications and surgical options. Curr Opin Pediatr 29: 80-86, 2017.

8. Hao YK, Zhang YK, Yang ZP, Li X, Yang Q and Li JM: The accuracy of magnetic resonance imaging in determining the osteotomy plane in osteosarcoma. Orthopedics 31: 544, 2008.

9. Kumta SM, Chow TC, Griffith J, Li CK, Kew J and Leung PC: Classifying the location of osteosarcoma with reference to the epiphyseal plate helps determine the optimal skeletal resection in limb salvage procedures. Arch Orthop Trauma Surg 119: 327-331, 1999.

10. Enneking WF, Springfield D and Gross M: The surgical treatment of parosteal osteosarcoma in long bones. J Bone Joint Surg Am 67: 125-135, 1985.

11. Skorupski KA, Uhl JM, Szivek A, Allstadt Frazier SD, Rebhun RB and Rodriguez CO Jr: Carboplatin versus alternating carboplatin and doxorubicin for the adjuvant treatment of canine appendicular osteosarcoma: A randomized, phase III trial. Vet Comp Oncol 14: 81-87, 2016.

12. Schwartz CL, Wexler LH, Krailo MD, Teot LA, Devidas M, Steinherz LJ, Goorin AM, Gebhardt MC, Healey JH, Sato JK, et al: Intensified chemotherapy with dexrazoxane cardioprotection in newly diagnosed nonmetastatic osteosarcoma: A report from the children's oncology group. Pediatr Blood Cancer 63: 54-61, 2016

13. Yu W, Tang L, Lin F, Yao Y and Shen Z: Pirarubicin versus doxorubicin in neoadjuvant/adjuvant chemotherapy for stage IIB limb high-grade osteosarcoma: Does the analog matter? Med Oncol 32: 307, 2015.

14. American Association of Tissue Banks: American Association of Tissue Banks provisional guidelines for cell, tissue and organ preservation: Reproductive council guidelines. Newsl Am Assoc Tissue Banks 4 (Suppl): S37-S40, 1980.

15. Tsuchiya H, Abdel-Wanis ME, Sakurakichi K, Yamashiro T and Tomita K: Osteosarcoma around the knee. Intraepiphyseal excision and biological reconstruction with distraction osteogenesis. J Bone Joint Surg Br 84: 1162-1166, 2002.

16. Cañadell J, Forriol F and Cara JA: Removal of metaphyseal bone tumours with preservation of the epiphysis. Physeal distraction before excision. J Bone Joint Surg Br 76: 127-132, 1994

17. Enneking WF, Dunham W, Gebhardt MC, Malawar M and Pritchard DJ: A system for the functional evaluation of reconstructive procedures after surgical treatment of tumors of the musculoskeletal system. Clin Orthop Relat Res (286): 241-246, 1993.

18. Tan PX, Yong BC, Wang J, Huang G, Yin JQ, Zou CY, Xie XB, Tang QL and Shen JN: Analysis of the efficacy and prognosis of limb-salvage surgery for osteosarcoma around the knee. Eur J Surg Oncol 38: 1171-1177, 2012.

19. Bi W, Wang W, Han G, Jia J and Xu M: Osteosarcoma around the knee treated with neoadjuvant chemotherapy and a custom-designed prosthesis. Orthopedics 36: e444-e450, 2013.

20. Houdek MT, Watts CD, Wyles CC, Rose PS, Taunton MJ and Sim FH: Functional and oncologic outcome of cemented endoprosthesis for malignant proximal femoral tumors. J Surg Oncol 114: 501-506, 2016

21. Ieguchi M, Hoshi M, Aono M, Takada J, Ohebisu N, Kudawara I and Nakamura $\mathrm{H}$ : Knee reconstruction with endoprosthesis after extra-articular and intra-articular resection of osteosarcoma. Jpn J Clin Oncol 44: 812-817, 2014

22. Hahn SB, Park HJ, Kim HS, Kim SH and Shin KH: Surgical treatment of malignant and aggressive bone tumors around the knee by segmental resection and rotationplasty. Yonsei Med J 44 $485-492,2003$
23. Campanacci L, Alì N, Casanova JM, Kreshak J and Manfrini M Resurfaced allograft-prosthetic composite for proximal tibial reconstruction in children: Intermediate-term results of an original technique. J Bone Joint Surg Am 97: 241-250, 2015.

24. Abdel-Ghani H, Ebeid W and El-Barbary H: Management of combined nonunion and limb-length discrepancy after vascularised fibular grafting. J Bone Joint Surg Br 92: 267-272, 2010

25. Cipriano CA, Gruzinova IS, Frank RM, Gitelis S and Virkus WW: Frequent complications and severe bone loss associated with the repiphysis expandable distal femoral prosthesis. Clin Orthop Relat Res 473: 831-838, 2015.

26. Sawamura C, Matsumoto S, Shimoji T, Ae K, Tanizawa $T$, Gokita T, Koyanagi $\mathrm{H}$ and Okawa A: Indications for and surgical complications of rotationplasty. J Orthop Sci 17: 775-781, 2012.

27. Forni C, Gaudenzi N, Zoli M, Manfrini M, Benedetti MG, Pignotti E and Chiari P: Living with rotationplasty-quality of life in rotationplasty patients from childhood to adulthood. J Surg Oncol 105: 331-336, 2012.

28. Dotan A, Dadia S, Bickels J, Nirkin A, Flusser G, Issakov J, Neumann Y, Cohen I, Ben-Arush M, Kollender Y and Meller I: Expandable endoprosthesis for limb-sparing surgery in children: Long-term results. J Child Orthop 4: 391-400, 2010.

29. Arteau A, Lewis VO, Moon BS, Satcher RL, Bird JE and Lin PP: Tibial growth disturbance following distal femoral resection and expandable endoprosthetic reconstruction. J Bone Joint Surg Am 97: e72, 2015

30. Abed YY, Beltrami G, Campanacci DA, Innocenti M, Scoccianti G and Capanna R: Biological reconstruction after resection of bone tumours around the knee: Long-term follow-up. J Bone Jt Surg Br 91: 1366-1372, 2009.

31. Bus MP, Dijkstra PD, van de Sande MA, Taminiau AH, Schreuder HW, Jutte PC, van der Geest IC, Schaap GR and Bramer JA: Intercalary allograft reconstructions following resection of primary bone tumors: A nationwide multicenter study. J Bone Joint Surg Am 96: e26, 2014.

32. Aponte-Tinao L, Ayerza MA, Muscolo DL and Farfalli GL: Survival, recurrence and function after epiphyseal preservation and allograft reconstruction in osteosarcoma of the knee. Clin Orthop Relat Res 473: 1789-1796, 2015.

33. DeGroot H III and Mankin H: Total knee arthroplasty in patients who have massive osteoarticular allografts. Clin Orthop Relat Res (373): 62-72, 2000.

34. Muscolo DL, Ayerza MA, Aponte-Tinao LA and Ranalletta M: Partial epiphyseal preservation and intercalary allograft reconstruction in high-grade metaphyseal osteosarcoma of the knee. J Bone Joint Surg Am 86: 2686-2693, 2004.

35. Karakaşli A, Satoğlu İS and Havitçioğlu H: A new intramedullary sustained dynamic compression nail for the treatment of long bone fractures: A biomechanical study. Eklem Hastalik Cerrahisi 26: 64-71, 2015

36. Bus MP, Dijkstra PD, van de Sande MA, Taminiau AH, Schreuder HW, Jutte PC, vander Geest IC, Schaap GR and Bramer JA: Intercalary allograft reconstructions following resection of primary bone tumors: A nationwide multicenter study. J Bone Joint Surg Am 96: e26, 2014.

37. Hornicek FJ, Gebhardt MC, Tomford WW, Sorger JI, Zavatta M, Menzner JP and Mankin HJ: Factors affecting nonunion of the allograft-host junction. Clin Orthop Relat Res 382: 87-98, 2001.

38. Faisham WI, Mat Saad AZ, Alsaigh LN, Nor Azman MZ, Kamarul Imran M, Biswal BM, Bhavaraju VM, Salzihan MS, Hasnan J, Ezane AM, et al: Prognostic factors and survival rate of osteosarcoma: A single-institution study. Asia Pac J Clin Oncol 13: e104-e110, 2017.

39. Ayerza MA, Farfalli GL, Aponte-Tinao L and Muscolo DL: Does increased rate of limb-sparing surgery affect survival in osteosarcoma? Clin Orthop Relat Res 468: 2854-2859, 2010. 